\title{
RESTOS DE Hippidion Y Equus (Amerhippus) PROCEDENTES DE LAS BARRANCAS DE SAN LORENZO, PLEISTOCENO TARDÍO (PROVINCIA DE SANTA FÉ, ARGENTINA)
}

\author{
José Luis PRADO y María Teresa ALBERDI \\ ${ }^{1}$ INCUAPA, Departamento de Arqueología. Universidad Nacional del Centro. \\ Del Valle 5737. B7400JWI Olavarría (Argentina). \\ e-mail:jprado@soc.unicen.edu.ar \\ ${ }^{2}$ Museo Nacional de Ciencias Naturales, CSIC. José Gutiérrez Abascal, 2. \\ 28006-Madrid (Spain).e-mail: malberdi@mncn.csic.es
}

Prado, J. L. \& Alberdi, M. T. 2008. Restos de Hippidion y Equus (Amerhippus) procedentes de las Barrancas de San Lorenzo, Pleistoceno tardío (Provincia de Santa Fé, Argentina). [Hippidion and Equus (Amerhippus) from the San Lorenzo cliff, late Pleistocene (Santa Fe province, Argentina).] Revista Española de Paleontología, 23 (2), 225-236. ISSN 0213-6937.

\begin{abstract}
Mammal fossil remains from Santa Fe Province (Argentina) are very poorly known. In this paper, best preserved specimens of Equidae (Mammalia, Perissodactyla) are presented. They come from the Pleistocene beds outcropping along the Río Paraná, near the city of San Lorenzo. The comparative study with other South American horse remains, mainly from Argentina and Brazil, allow their identification as Equus (Amerhippus) neogeus and Hippidion principale. The stratigraphical sequence lacks paleomagnetic or radiometric data, but based on stratigraphic correlation the fossiliferous level was placed between $128 \mathrm{ka}$ (isotopic stage 5e) of the and $28 \mathrm{ka}$ (isotopic stage 2e).
\end{abstract}

Key words: Equidae, Hippidion, Equus (Amerhippus), Latest Pleistocene, Santa Fe, Argentina.

\section{RESUMEN}

Los mamíferos fósiles de la Provincia de Santa Fé (Argentina) son escasamente conocidos. En este trabajo, se describen los restos mejor conservados de équidos (Mammalia, Perissodactyla) encontrados en los niveles pleistocenos aflorantes a lo largo del Río Paraná, cerca de la ciudad de San Lorenzo. El estudio comparativo con otros caballos provenientes de distintas localidades de América del Sur, en su mayoría argentinas y brasileñas, permite identificarlos como Equus (Amerhippus) neogeus e Hippidion principale. La sección estratigráfica analizada no cuenta con dataciones radiométricas o estudios paleomagnéticos que permitan calibrar con cierta precisión la edad de los depósitos portadores de équidos. En consecuencia, en base a correlaciones estratigráficas, los restos hallados estarían situados en un lapso que se extiende desde 128 ka (inicio del Pleistoceno Superior, estadio isotópico 5e) como edad máxima hasta momentos anteriores al comienzo del estadio isotópico 2e (28 ka).

Palabras claves: Equidae, Hippidion, Equus (Amerhippus), Pleistoceno tardío, Santa Fé, Argentina.

\section{INTRODUCCIÓN}

En América del Sur, la familia Equidae se encuentra representada por dos géneros: Equus (Amerhippus) Hoffestetter, 1950 e Hippidion Owen, 1869. En general, ambos géneros presentan especies adaptadas a los ambientes de montaña y llanura. Este grupo fue revisado por Alberdi \& Prado (1993) y Prado \& Alberdi (1994) quienes indicaron la presencia de tres especies distintas de Hippidion y cinco de Equus (Amerhippus).
En el continente americano, los équidos se extinguieron hacia fines del Pleistoceno y fueron reintroducidos recientemente en tiempos históricos. Los registros de caballos en el centro y el norte de Argentina, al igual que otros grupos de mamíferos, son verdaderamente escasos (Alberdi et al., 2008). La mayoría de los datos que sustentan las distintas hipótesis sistemáticas y paleobiogeográficas provienen de la región pampeana y de la región patagónica. Los escasos hallazgos de megafauna cuaterna- 
ria para la provincia de Santa Fé se remontan a los citados por Darwin (1846), Ameghino (1889), De Carles (1912), Frenguelli $(1928,1932)$ y Castellanos (1924). Recientemente, Zurita et al. (2002) estudiaron los mamíferos del arroyo El Tiapalito (Reconquista, Santa Fé) y Miño Boilini et al. (2006) han descrito un cráneo de Toxodon del Lujanense de Santa Fé.

El objetivo principal de este trabajo es el estudio sistemático de los restos de équidos procedentes de las barrancas del río Paraná, realizando una comparación morfométrica con los datos existentes de otras localidades en América del Sur (Alberdi et al., 1986, 1987, 1989, 2001 a, b, 2003, 2006, 2007; Ubilla \& Alberdi, 1990; Alberdi \& Prado, 1993, 2004; Prado \& Alberdi, 1994; Prado et al., 1998, 2005; Alberdi \& Prieto, 2000; Alberdi \& Frassinetti, 2000; Rincón et al., 2006).

\section{DESCRIPCIÓN DEL ÁREA ESTUDIADA}

El Puerto San Martín (32 44’29” S, 60 44’ 42” O) se encuentra ubicado en las proximidades de la localidad de San Lorenzo a $23 \mathrm{~km}$ al Norte de la Ciudad de Rosario, en la orilla occidental del río Paraná (Fig. 1). En la figura 2 se

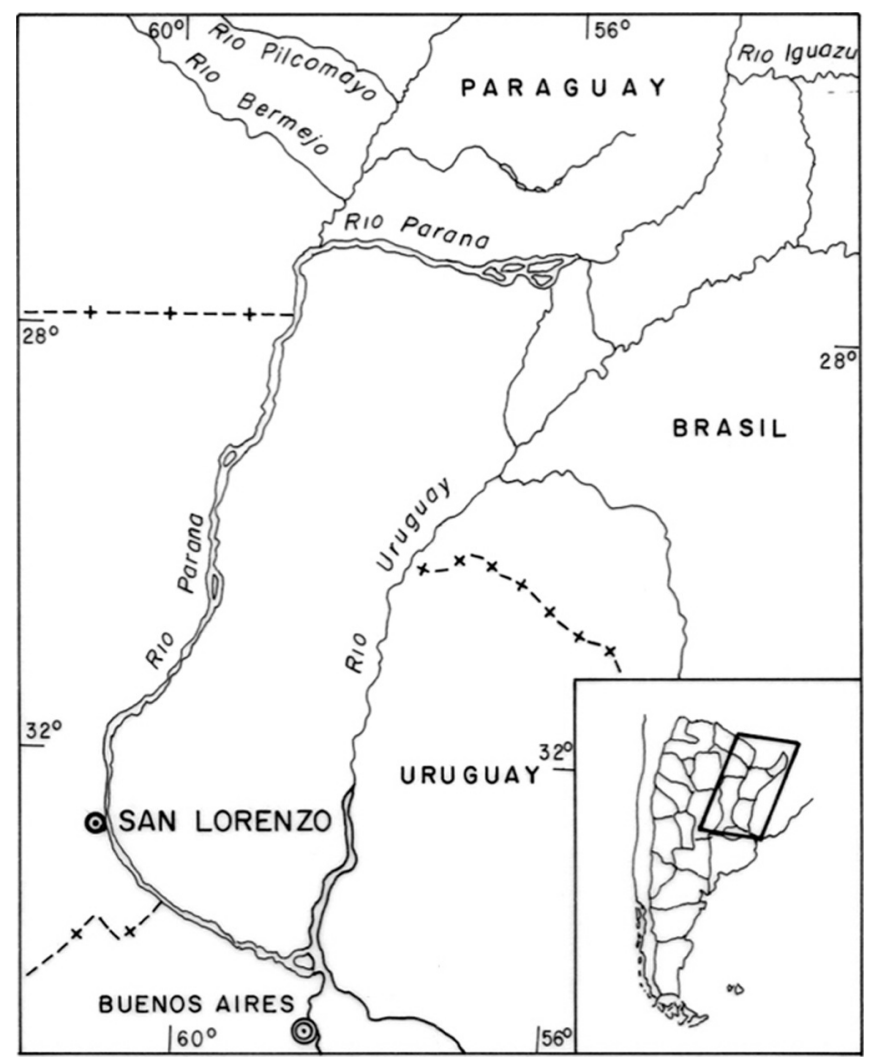

Figura 1. Situación geográfica de San Lorenzo, en las barrancas del Paraná (Santa Fé, Argentina).

Geographical location of the Paraná cliffs (Santa Fe province, Argentina). presenta un perfil esquemático con la situación de los fósiles recuperados de las barrancas del río Paraná.

Este río conforma conjuntamente con los ríos Paraguay y Uruguay el sistema fluvial del río de La Plata. La cuenca del río Paraná cubre una superficie de $1,510.000 \mathrm{~km}^{2}$, en la cual se identifican tres sectores: el Paraná Superior (de la naciente a la confluencia con el río Paraguay), el Paraná Medio (de la confluencia hasta el ápice del delta) y el Paraná Inferior (correspondiente al propio delta).

Geológicamente, Iriondo (1991) detalla que el río Paraná atraviesa en forma perpendicular o diagonal varios bloques tectónicos, lo que produce la aparición de segmentos con distintas orientaciones y pendientes locales. Dichos bloques, que tienen origen en las actividades tectónicas del Holoceno

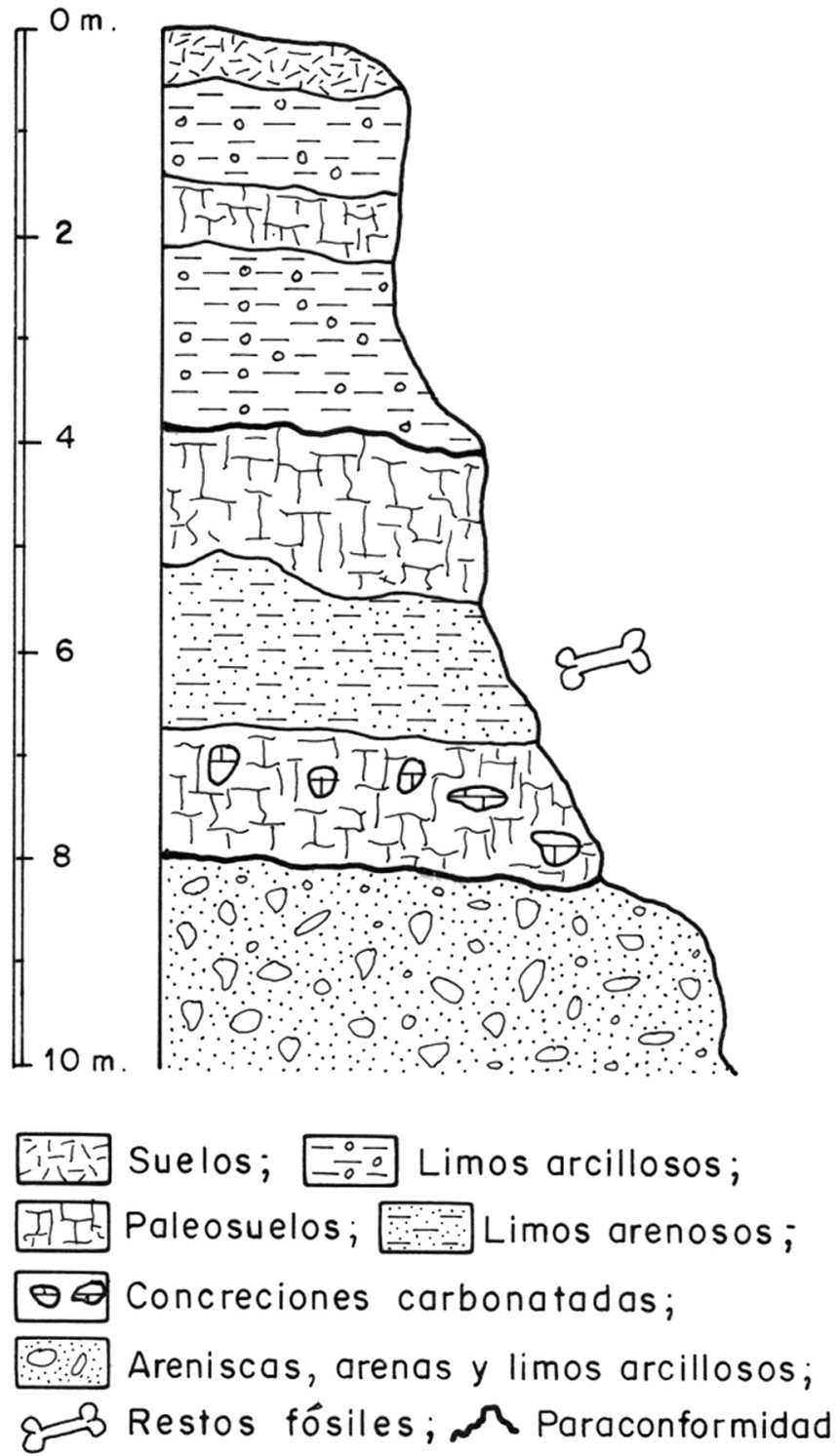

Figura 2. Perfil estratigráfico de las barrancas del río Paraná (Santa Fé, Argentina) con indicación del nivel fosilífero. Stratigraphic profile of the Parana river cliffs with fossiliferous level (Santa Fe province, Argentina). 
y también probablemente en la actualidad, ocasionan diferencias en las altitudes de los diferentes sectores, variando entre 2 y 5 metros.

Los sedimentos pleistocenos que afloran en las márgenes de río Paraná en el área de San Lorenzo (denominados corrientemente "sedimentos pampeanos") presentan dificultades para su clasificación y correlación debido principalmente a su homogeneidad litológica. Esto ya había sido mencionado por Castellanos (1938), quien describió originalmente la sucesión sedimentaria del Sur de la provincia de Santa Fé basándose en información de perforaciones y cortes naturales en la barranca del Río Paraná en la ciudad Rosario.

Originalmente, d'Orbigny (1842-44) utilizó el término "Argille pampéene" (non vidum sensu Tonni et al., 1999) para nombrar los sedimentos marrón-rojizos aflorantes cerca del Río de la Plata. Luego, los términos "Formación Pampeana", "Formación Pampiano" y "sedimentos pampeanos", entre otros, fueron derivados de este nombre original (Fidalgo et al., 1975). Los problemas emergentes de clasificación y correlación que genera esta aparente homogeneidad litológica interregional fueron claramente indicados y discutidos por González Bonorino (1965).

En la provincia de Buenos Aires, bajo esta denominación, se agrupa a las formaciones Ensenada y Buenos Aires; las dos unidades son muy similares y en algunos casos de difícil separación, por lo que actualmente a este conjunto se lo agrupa en general como sedimentos pampeanos, atribuyéndolos a los Piso/Edad Ensenadense y Bonaerense respectivamente. La primera fue referida en la provincia de Buenos Aires a la biozona de Tolypeutes pampaeus y la segunda a Megatherium americanum por Cione \& Tonni (1999) y Tonni et al. (1999). Abarcan gran parte de la llanura Chaco-Pampeana, son depósitos medianos a finos, limos y arcillas con intercalaciones calcáreas concrecionales o tipo mantiformes (tosca). El color dominante es el castaño, con tonalidades amarillentas a rojizas. En gran parte corresponden a sedimentos transportados por el viento desde la cordillera ya emergida para esa época y desde los llanos secos y poco vegetados que se formaron hacia el oeste de la misma. Inmensas nubes de polvo y trizas vítreas (cenizas volcánicas) de los volcanes cordilleranos alcanzaron a depositarse hasta el Atlántico, dando origen al conocido loess pampeano que fue retrabajado incesantemente por ríos, arroyos y pequeños cursos de agua, redepositándose en cauces y planicies costeras hasta la actualidad.

Recientemente, se propuso la Formación Rosario (Parent et al., 2002) para denominar los 20 a 30 metros de limos marrón-rojizos a marrón-amarillentos con arcillas verdes intercaladas que se asientan sobre arenas finas laminadas que ocurren conspicuamente a lo largo de las barrancas del Río Paraná (denominadas localmente "Ensenadense medio e inferior" por Castellanos, 1943). Por debajo de estos niveles de arenas, se encuentra la formación Puelches (Plioceno) que es de origen fluvial y está integrada por arenas muy finas, hasta pulverulentas, tendiendo a granulometría mediana hacia la base.
Parent et al. (2002) identifican en la Formación Rosario cinco conjuntos o secuencias limo marrón-arcilla verde que denotan cambios climáticos periódicos bien definidos. El nivel S1 corresponde al Ensenadense superior y Belgranense inferior de Castellanos (1938, 1943); el nivel S2 corresponde al Belgranense medio y superior; el S3 corresponde al Bonaerense inferior y medio; el S4 al Bonaerense superior y el S5 al Lujanense y Platense inferior de Castellanos (Parent et al., 2002).

\section{MATERIAL Y MÉTODO}

Los materiales estudiados se encuentran depositados en las colecciones del Museo de Historia y Galería de Arte del Centro Cultural de San Lorenzo (provincia de Santa Fé, Argentina) y fueron recuperados por el Sr. Pedro Mondoni, vecino de la localidad. Estos se reducen a un cráneo incompleto (núm. 161) y un metatarsiano derecho del tercer dedo (núm. 130) de Hippidion y un fragmento de mandíbula (núm.135) de Equus (Amerhippus). Tanto los restos del cráneo como la mandíbula los hemos comparado morfológica y biométricamente con otros ejemplares procedentes de varias localidades de América del Sur depositados en distintos museos e instituciones. Para su descripción y análisis morfométrico, hemos seguido la nomenclatura y normas recomendadas en la "Hipparion Conference" (Eisenmann et al., 1988), así como los estudios previos realizados por Alberdi \& Prado $(1992,1993)$ y Prado \& Alberdi (1994). Todas las dimensiones están expresadas en milímetros. A fin de determinar sistemáticamente el MTIII, se realizó un análisis discriminante con los restos del esqueleto apendicular de las distintas especies del género Hippidion estudiados por Alberdi \& Prado (1993).

\section{PALEONTOLOGÍA SISTEMÁTICA}

Orden PERISSODACTYLA Owen, 1848

Familia Equidae Gray, 1821

Subfamilia Equinae Gray, 1821

Género Equus Linnaeus, 1758

Subgénero Equus (Amerhippus) Hoffstetter, 1950

\section{Equus (Amerhippus) neogeus Lund, 1840} Fig. 3

Material. Una mandíbula incompleta (núm. 135) de San Lorenzo (provincia de Santa Fé, Argentina) que conserva la sínfisis sin incisivos ni caninos y la rama derecha prácticamente entera.

Descripción: La mandíbula de Equus (Amerhippus) neogeus es robusta y en la superficie oclusal de los dientes inferiores se observa que el doble bucle (lazo: metacónido-metastílido) es redon- 
deado y angular, respectivamente (Fig. 3). El linguafléxido es somero y más angular sobre P3-P4 y más abierto sobre M1-M2. El ectofléxido varía desde profundo a somero y sólo en algunos M3 entra en contacto con el linguafléxido.

A este ejemplar le falta parte de la sínfisis y del borde basal, sin embargo es el ejemplar más completo de una mandíbula de Equus (Amerhippus) neogeus encontrada hasta ahora en la provincia de Santa Fé. La sínfisis y el cuerpo mandibular derecho tienen un aspecto robusto, conserva los alveolos de los caninos y los incisivos. En la hemimandíbula derecha se conservan los dientes yugales p3-m3, falta p2. Sus dimensiones están en la Tabla 1. Esta hemimandíbula conserva la rama ascendente. Esta dentición corresponde a un animal viejo con la superficie oclusal de los dientes bastante desgastada, empezando a perderse las figuras de la superficie oclusal. El preflexido y postflexido se encuentra re- ducido en p3, m1 y m2, el doble-nudo muy abierto en general y desapareciendo en $\mathrm{m} 1 \mathrm{y} \mathrm{m} 2$. El ectofléxido es profundo sin atravesar el istmo en p3-p4-m1 y atravesándolo sin entrar en contacto con el linguaflexido en $\mathrm{m} 2 \mathrm{y} \mathrm{m} 3$, el cual es amplio y somero. Las dimensiones de la mandíbula se han tomado siguiendo a Eisenmann et al. (1988): $1=447,2=112,8,3=91 \mathrm{ca}, 4=88,2,5$ $=180 \mathrm{ca}$ (falta p2), $6=145,7,7=67,8=>257$ (borde posterior alterado), $9=>230,10=>140,13=85 \mathrm{ca}$.

Discusión: $E$. (A.) neogeus es la especie más grande y más grácil de todas la especies de Equus (Amerhippus) de América del Sur. Se caracteriza por tener el cráneo grande y las zonas preorbital y nasal alargadas. Los huesos de las extremidades son grandes, robustos y los más gráciles de las es-

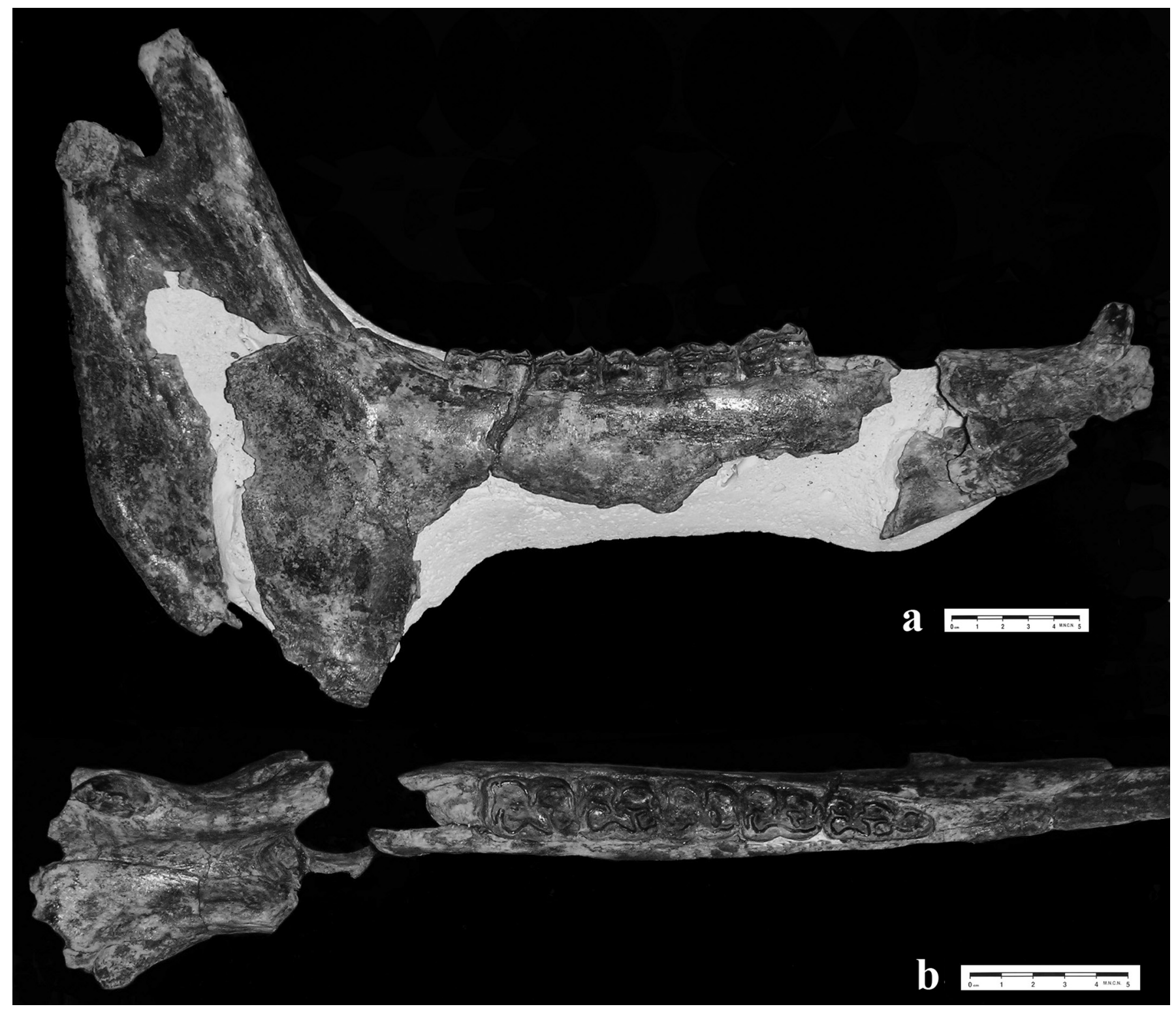

Figura 3. Hemimandíbula derecha de Equus (Amerhippus) neogeus Lund, 1840 en vista lateral externa (a) y en vista oclusal (b), respectivamente procedente de San Lorenzo, Puerto General San Martín, bajada del Quebracho (Santa Fé, Argentina). Equus (Amerhippus) neogeus Lund, 1840 right mandible in lateral (a) and occusal (b) views from San Lorenzo (General San Martín Port, Quebracho slope, Santa Fe, Argentina). 


\begin{tabular}{c|ccccc} 
serie der Hippidion & Ls & As & H & Lpr & \\
\hline P1 & 20 & 10 & & & \\
P2 & 43,1 & 31,5 & $44 \mathrm{ca}$ & 7,8 & \\
P3 & 34 & 37,6 & $>50$ & 11,6 & \\
P4 & 32 & 36 & & 10,5 & \\
M1 & 31,2 & 36,5 & & 10 & \\
M2 & 29,6 & 32 & & 10,9 & \\
M3 & 32,6 & 28 & & 11,5 & \\
\hline serie izq Hippidion & Ls & As & H & Lpr & \\
\hline P3 & 34,8 & 35,5 & & 10 & \\
P4 & 32,6 & 36 & & 10 & \\
M1 & 32 & 36 & & & \\
M2 & 30 & 33 & & 11 & \\
M3 & 32,5 & 27,5 & & 9,2 & \\
\hline serie der Equus (Amerhippus) & Ls & As & H & posflex. & lazo \\
\hline p3 & 28 & 19,8 & & 7 & 16,5 \\
p4 & 26,5 & 20 & & 10 & 16 \\
m1 & 26,2 & 19 & & 2,8 & 14,5 ca \\
m2 & 26 & 17,5 & & 6,8 & 13,8 \\
m3 & 35 & 14 & & 8,8 & 13 \\
\hline & & & &
\end{tabular}

Tabla 1. Dimensiones en milímetros de los dientes superiores del cráneo de Hippidion principale (Lund, 1846); y dientes inferiores de la mandíbula de Equus (Amerhippus) neogeus Lund, 1840 procedentes de San Lorenzo, Puerto General San Martín, bajada del Quebracho (Santa Fé,Argentina). Ls, longitud medio-distal en superficie oclusal; As, anchura linguo-bucal en superficie ocusal; $\mathrm{H}$, altura del diente; Lpr, Longitud mesio-distal del protocono en superficie oclusal; postflex., longitud mesiodistal del postfléxido; lazo, longitud del lazo metacónido-metastílido en superficie oclusal.P/p, premolares superiores/inferiores; $\mathrm{M} / \mathrm{m}$, molares superiores/inferiores Upper cheek teeth measurements of Hippidion principale (Lund, 1846) (cranium) and lower teeth of Equus (Amerhippus) neogeus Lund, 1840 (mandible) from San Lorenzo (General San Martín Port, Quebracho slope, Santa Fe province). Ls, mesio-distal length in occlusal surface; As, bucco-lingual breadth in occlusal surface; $H$, height; Lpr, mesio-distal length of protocone in occlusal surface; postflex., mesio-distal length of postflexid; double-knot, length of the metaconid-metastylid in occlusal surface; $P / p$, upper/lower premolars, $\mathrm{M} / \mathrm{m}$, upper/lower molars. Dimensions in millimeters.

pecies de Equus (Amerhippus) conocidas en América del Sur. Tanto la morfología de los dientes como las dimensiones de esta mandíbula presentan las características de los restos conocidos de E. (A.) neogeus de otras localidades de América del Sur. Su comparación con la única otra mandíbula existente, más o menos completa, de esta especie procedentes de la provincia de Buenos Aires (ZMK-238 procedente de Arroyo Ramallo) indica que este ejemplar es algo más gran- de, sobre todo en los molares (Tabla 2). Ambos ejemplares corresponden a individuos muy viejos e incompletos.

Género Hippidion Owen, 1869

$$
\begin{gathered}
\text { Hippidion principale (Lund, 1846) } \\
\text { Figs 4, } 5
\end{gathered}
$$

Material: Los restos recuperados en las barrancas del área de San Lorenzo se reducen a un cráneo que le falta la parte distal (núm. 161) y un metatarsiano derecho del tercer dedo completo (núm. 130).

Descripción: El cráneo está incompleto y algo deformado, de aspecto robusto, y carece de fosa preorbital, si bien se observa una depresión en dicha zona (Fig. 4). El perfil dorsal del cráneo en vista lateral presenta una convexidad anterior y la parte de la inflexión fronto-nasal falta prácticamente entera por lo que la vista dorso-occipital se desconoce en este ejemplar. El hueso nasal esta roto y su hendidura está bastante retraída alcanzando el nivel del mesostilo de M3. La cresta facial hace saliente a nivel posterior al parastilo de M1 en el lado derecho. La órbita se conserva entera en el lado derecho y sólo la parte anterior en el lado izquierdo. En vista ventral, se observa que el paladar está muy deteriorado y no se puede observar el vomer. La sínfisis es estrecha y alargada. Los dientes yugales superiores están relativamente curvados en sentido linguo-ventral, con morfología oclusal primitiva, tipo Pliohippus, característica de este género. La sínfisis alargada conserva todos los incisivos y caninos que son robustos, podría corresponder a un individuo macho. Las series yugales están bastante completas, P1-M3 derechos y P3-M3 izquierdos. Los premolares son más robustos que los molares, y tienen los estilos también más pronunciados que los molares; los protoconos tienen forma oval alargada. La morfología dental varía en relación con el grado de desgaste y éste en relación a la edad de los animales. Las dimensiones de los dientes están en la Tabla 1. Toda la dentición parece estar en un desgaste medio, lo cual representa una edad adulta para el individuo al que corresponden estos restos. Las únicas dimensiones que se pueden tomar de este ejemplar son: $7=109,8=94$ el derecho y 94, 2 el izquierdo, $9=197,5$ (incluido $\mathrm{P} 1=210$ ), $14=45,5,15=63$, $18=206 \mathrm{ca}, 23=458,26=145 \mathrm{ca}, 28=74,6,29=52,5,36=$ 73 , la distancia entre los dos I3 = 72, el diastema entre P1 y I1 derecho $=126$, el diastema entre P1- C $=67$, y entre $\mathrm{C}$ y I1 = 43 (ver Tabla 3). Desde un punto de vista comparativo, las dimensiones de este cráneo indican que se encuentra entre los más grandes de la especie. El hecho de que este cráneo esté bastante incompleto así como que el número de cráneos completos conocidos de este género, en general, y de esta especie, en particular, sea bastante reducido impide llevar a cabo un análisis estadístico comparativo de sus dimensiones.

El MTIII derecho es un ejemplar completo y relativamente corto y ancho, con un índice de gracilidad característico del género Hippidion ( $\mathrm{IG}=16,13$ ) (Fig. 5). Las dimensiones están en la Tabla 4. La morfología de la extremidad proximal está algo alterada, con la articulación para el cuboides rota, y la articulación distal se conserva bien con la anchura supra-articular muy pronunciada. La huella del MtIV sobrepasa la mitad de la longitud del tercer dedo, o el punto de diáfisis, mientras que la huella del MTII parece quedar más arriba (Fig. 5). 


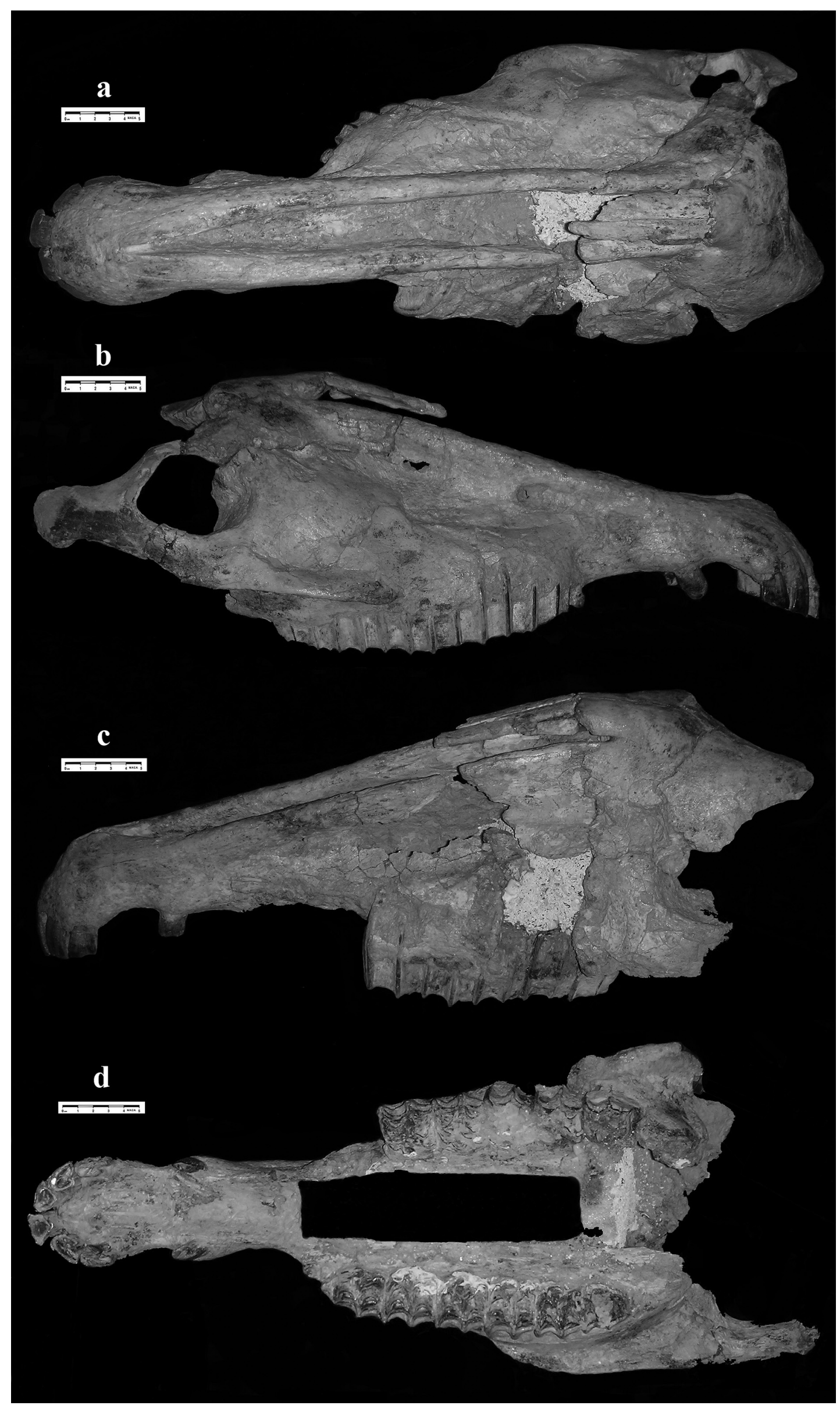




\begin{tabular}{|c|c|c|c|c|c|c|c|c|c|c|c|c|c|c|c|}
\hline sigla & localidad & med 1 & med 2 & med 3 & med 4 & med 5 & med 6 & med 7 & med 8 & med 9 & med 10 & med 11 & med 12 & med 13 & med 14 \\
\hline ZMK-238 & Arroyo Ramallo & 460 & 132 & 86,7 & 82,8 & 168,5 & 148 & 62,1 & 248 & 234 & 127 & 96,9 & 73 & 97,5 & 46,4 \\
\hline núm. 135 & San Lorenzo & 447,2 & 112,8 & $91 \mathrm{ca}$ & 88,2 & $180 \mathrm{ca}$ & $145,7 \mathrm{ca}$ & 67,8 & $>257$ & $>230$ & $>140$ & & & $85 \mathrm{ca}$ & \\
\hline
\end{tabular}

Tabla 2. Dimensiones en milímetros de la mandíbula de Equus (Amerhippus) neogeus Lund, 1840 procedente de San Lorenzo, Puerto General San Martín, bajada del Quebracho (Santa Fé, Argentina), comparada con la mandíbula ZMK-238 de E. (A.) neogeus procedente de Arroyo Ramallo (provincia de Buenos Aires, Argentina) y depositada en el Zoologisk Museum (Copenhague, Dinamarca). Los números (med) corresponden a las distintas medidas recomendadas en la "Hipparion Conference", celebrada en Nueva York en 1981 (Eisenmann et al., 1988), todas las dimensiones están expresadas en milímetros. Mandible measurements of Equus (Amerhippus) neogeus Lund, 1840 from San Lorenzo (General San Martín Port, Quebracho slope, Santa Fe province) compared with ZMK-238 mandible of E. (A.) neogeus from Arroyo Ramallo (Buenos Aires province, Argentina) and stored at Zoologisk Museum (Copenhague, Dinamarca). Numbers (med) correspond to recommended measures of the "Hipparion Conference", New York, 1981 (Eisenmann et al., 1988). All dimensions are expressed in millimetres.

Discusión: A pesar de los escasos restos de Hippidion estudiados, el enorme tamaño de la parte del cráneo que se conserva y su comparación con otros restos craneales de $H$. principale, encontrados en otras localidades de América del Sur, y la morfología y dimensiones de sus molares permiten identificar claramente la presencia de Hippidion principale en esta localidad.

En el caso del MTIII derecho, se incluyó en el análisis discriminante elaborado con 35 ejemplares de MTIII de Hippidion procedentes de distintas localidades de América del Sur (datos de Alberdi y Prado), quedando claramente incluido entre los restos de $H$. principale. Concretamente, si utilizamos el método de la introducción de las variables independientes juntas, los resultados del discriminante indican que la clasificación es correcta en el $100 \%$ de los casos; aplicándole la validación cruzada, este acierto alcanza el 93,3\% de los casos; si le aplicamos como estadístico el método de inclusión por pasos, distancias de Mahalanobis, los resultados indican una clasificación correcta en el 90,9\% de los casos y la validación cruzada alcanza un $87,9 \%$ de los casos correctamente identificados (Fig. 6).

\section{DISCUSIÓN Y CONCLUSIONES}

El nombre de Hippidion, creado por Owen en 1869, junto con el de Equus, han sido citados en numerosas localidades de Argentina y otros países de América del Sur. El primer representante de Hippidion fue descrito a partir de un único molar de una brecha antigua de la Cueva de Lagoa Santa (Brasil) y figurado por Lund en 1846. Alberdi \& Prado (1993) y Prado \& Alberdi (1996) consideran que este género es endémico en América del Sur donde se expande desde el Plioceno superior al Pleistoceno final

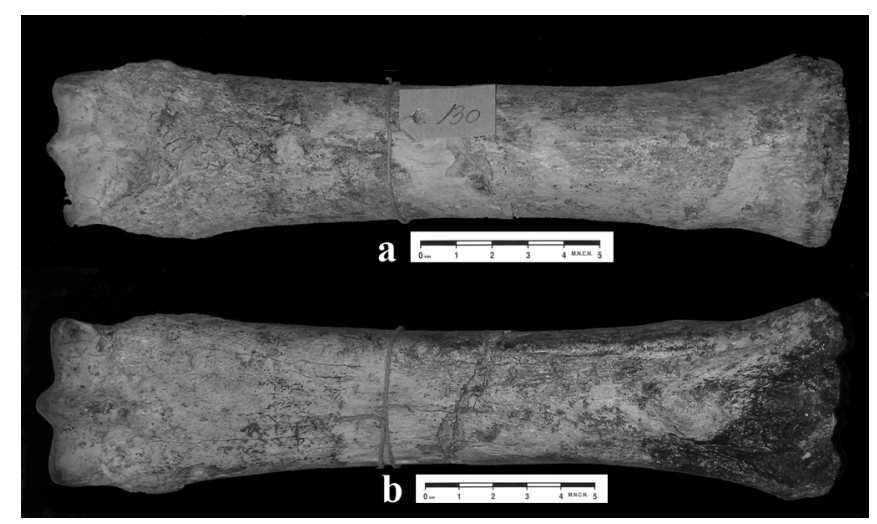

Figura 5. Metatarsiano derecho (MTIII) del tercer dedo de Hippidion principale (Lund, 1846) en vista anterior (a) y posterior (b) procedente de San Lorenzo, Puerto General San Martín, bajada del Quebracho (Santa Fé, Argentina). Anterior (a) and posterior (b) views of right metatarsian (MCIII) of Hippidion principale (Lund, 1846) from San Lorenzo (General San Martín Port, Quebracho slope, Santa Fe, Argentina).

(Alberdi \& Prado, 1993) y geográficamente se distribuye en gran parte de América del Sur, principalmente en Argentina, Bolivia, Brasil, Chile, Colombia, Perú y Uruguay. El primer representante de Equus fue recuperado por Darwin en Bahía Blanca durante la expedición del Beagle y descrito por Owen (1840).

La revisión taxonómica de los géneros Hippidion y Equus (Amerhippus) fue llevada a cabo por Alberdi \& Prado (1993) y Prado \& Alberdi (1994), respectivamente, mediante análisis multivariantes sobre variables cuantitativas de los restos más significativos del esqueleto apendicular. Estos análisis indicaron la presencia de tres grupos distin-

Figura 4. Cráneo de Hippidion principale (Lund, 1846) en vista dorsal (a), lateral derecha (b), lateral izquierda (c) y en vista ventral (d), respectivamente procedente de San Lorenzo, Puerto General San Martín, bajada del Quebracho (Santa Fé, Argentina). Hippidion principale (Lund, 1846) cranium in dorsal (a), lateral right (b), lateral left (c) and ventral (d) views from San Lorenzo (General San Martín Port, Quebracho slope, Santa Fe, Argentina). 


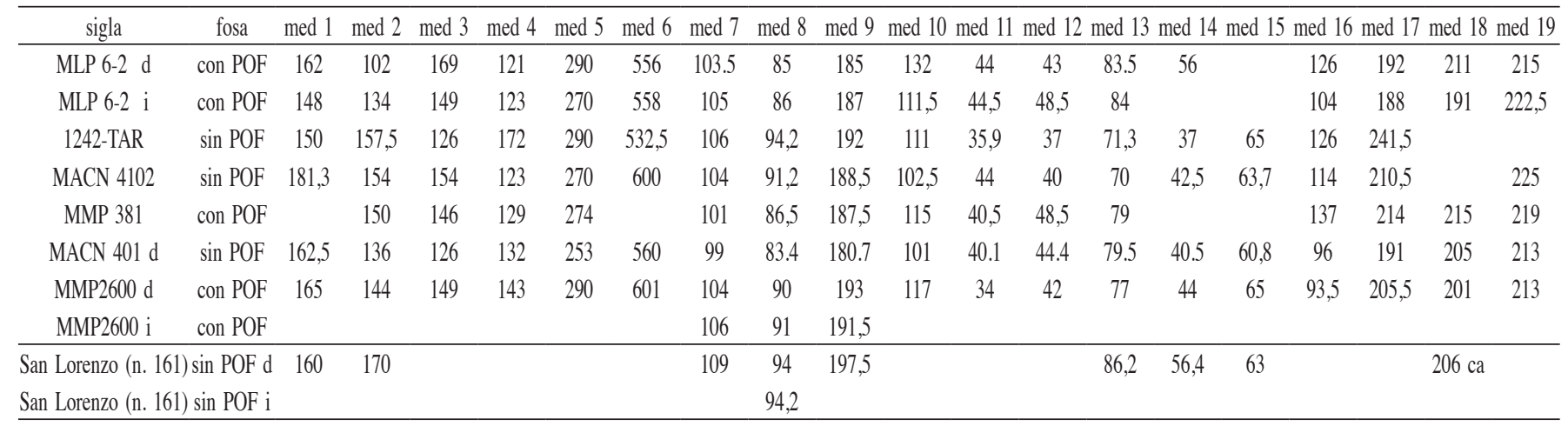

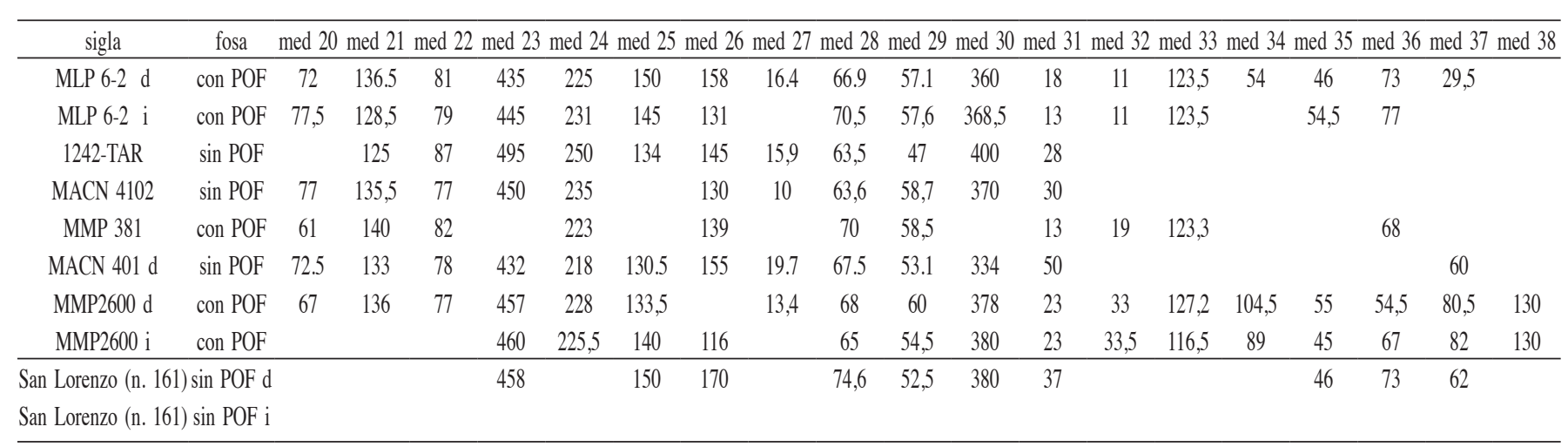

Tabla 3. Dimensiones en milímetros del cráneo de Hippidion principale (Lund, 1846) procedente de San Lorenzo, Puerto General San Martín, bajada del Quebracho (Santa Fé, Argentina) comparado con otros cráneos procedentes de la provincia de Buenos Aires. Concretamente: MMP-2600, cráneo descrito por Alberdi et al. (2001b) depositado en el Museo de Ciencias Naturales "Lorenzo Scaglia" de Mar del Plata; MLP 6-2, cráneo descrito por Moreno (1891), depositado en el Museo de La Plata; 1242, cráneo de Tarija depositado en la Universidad de La Paz (Bolivia); MCN-4102, cráneo de Arrecife y MCN-401 de Luján, depositados en el Museo Nacional de Ciencias Naturales "Bernardino Rivadavia”; y MMP-381, cráneo descrito por Reig (1957) como Onohippidion sp., de Baliza Chica a pocos kilómetros al N de Miramar y depositado en el Museo de Ciencias Naturales "Lorenzo Scaglia" de Mar del Plata.

Los números corresponden a las normas de nomenclatura recomendadas en la "Hipparion Conference", celebrada en Nueva York en 1981 (Eisenmann et al., 1988), todas las dimensiones están expresadas en milímetros. Abreviaturas: POF, fosa preorbital; d, derecha; i, izquierda.

Skull measurements of Hippidion principale (Lund, 1846) from San Lorenzo (General San Martín Port, Quebracho slope, Santa Fe province) compared with: MMP-2600, the skull described by Alberdi et al.(2001b) from the Mar del Plata sea-cliffs, stored at the Museo de Ciencias Naturales "Lorenzo Scaglia” of Mar del Plata; MLP 6-2, the skull described by Moreno (1891) from Loberia, stored at the Museo de La Plata; 1242, the skull from Tarija stored at the La Paz University (Bolivia); MCN-4102, skull from Arrecife and MCN-401 skull from Luján, stored at the Museo Nacional de Ciencias Naturales "Bernardino Rivadavia"; and MMP-381, skull described by Reig (1957) as Onohippidion sp., from Baliza Chica close to N Miramar, stored at the Museo de Ciencias Naturales "Lorenzo Scaglia" of Mar del Plata. Numbers correspond the the nomenclature recommendatios of the "Hipparion Conference", New York, 1981 (Eisenmann et al., 1988). All dimensions are expressed in millimetres. Abbreviatures: POF, preorbital fossa; $d$, right; $i$, left.

tos, en el caso del género Hippidion, y cinco en el caso de Equus (Amerhippus). Los primeros fueron asignados a las tres especies válidas que ellos determinan: Hippidion devillei (Gervais, 1855), Hippidion principale (Lund, 1846) e Hippidion saldiasi (Roth, 1899). En el caso de Equus (Amerhippus), fueron agrupados en cinco especies distintas: Equus (Amerhippus) neogeus Lund, 1840, Equus (Amerhippus) insulatus C. Ameghino (en F. Ameghino 1904), Equus (Amerhippus) santaeelenae (Spillmann, 1938), Equus (Amerhippus) andium (Branco, 1883) у Equus (Amerhippus) lasallei Daniel, 1948; posteriormente descrita y figurada en detalle por Porta (1960). Esta última se mantuvo a nivel específico dada la gran envergadura de la única pieza existente y la imposibilidad de llevar a cabo una contrastación estadística de la misma.

A la vista de los datos existentes y las discusiones previas, podemos identificar los restos de équidos procedentes del área de río Paraná, en el entorno de San Lorenzo, como Equus (Amerhippus) neogeus e Hippidion principale.

Esta asociación no es frecuente. La presencia de ambos géneros simultáneamente se ha citado en la Formación Sopas de Uruguay (Ubilla et al., 2004), en niveles referidos 


\begin{tabular}{|c|c|c|c|c|c|c|c|c|c|c|c|c|c|c|}
\hline Localidad & med 1 & med 2 & med & med 4 & med 5 & med 6 & med 7 & med 8 & med 9 & med 10 & med 11 & med 12 & med 13 & med 14 \\
\hline San Lorenzo & 248 & 239 & 40 & 35 & 59 & 47 & 53 & 13 & 7 & 56 & 51,2 & 40 & 29,6 & 34,5 \\
\hline Taguatagua & 252 & 245 & 38 & 33 & 59 & 42 & 54 & 12,5 & 9,9 & 56,6 & 52,7 & 39 & 29,6 & 34,5 \\
\hline Tarija & 248 & 238,5 & 38 & 37,4 & 60 & 46 & 55 & 11 & 10 & 60,5 & 55,7 & 39 & 31 & 36,2 \\
\hline Tarija & 240,5 & 231 & 40,6 & 38 & 59 & 45,5 & 50 & 11,3 & 9 & 59,4 & 52,7 & 37,9 & 31,2 & 34,7 \\
\hline Tarija & 239 & 231 & 38 & 32,8 & 56 & 41 & 50,6 & 10 & 6,6 & 53,3 & 50,4 & 38 & 29,4 & 34,5 \\
\hline Tarija & 235 & 226 & 37,3 & 32,2 & 55,2 & 39,7 & 47,2 & 10,1 & 6,8 & 52,4 & 49,2 & 38,5 & 29 & 32,3 \\
\hline
\end{tabular}

Tabla 4. Dimensiones (en mm) del MTIII de Hippidion principale (Lund, 1846) de San Lorenzo (Puerto General San Martín, bajada del Quebracho, provincia de Santa Fé, Argentina) comparado con algunos otros restos de América del Sur de esta especie. Los números indican la nomenclatura recomendada en la "Hipparion Conference" (Eisenmann et al., 1988). Todas las dimensiones están en milímetros.

Measurements of the MTIII of Hippidion principale (Lund, 1846) from San Lorenzo (General San Martín Port, Quebracho slope, Santa Fe province) compared with other metatarsals of this species from South American localities. Codes following the "Hipparion Conference" (Einsenmann et al., 1988). All dimensions are expressed in millimetres.

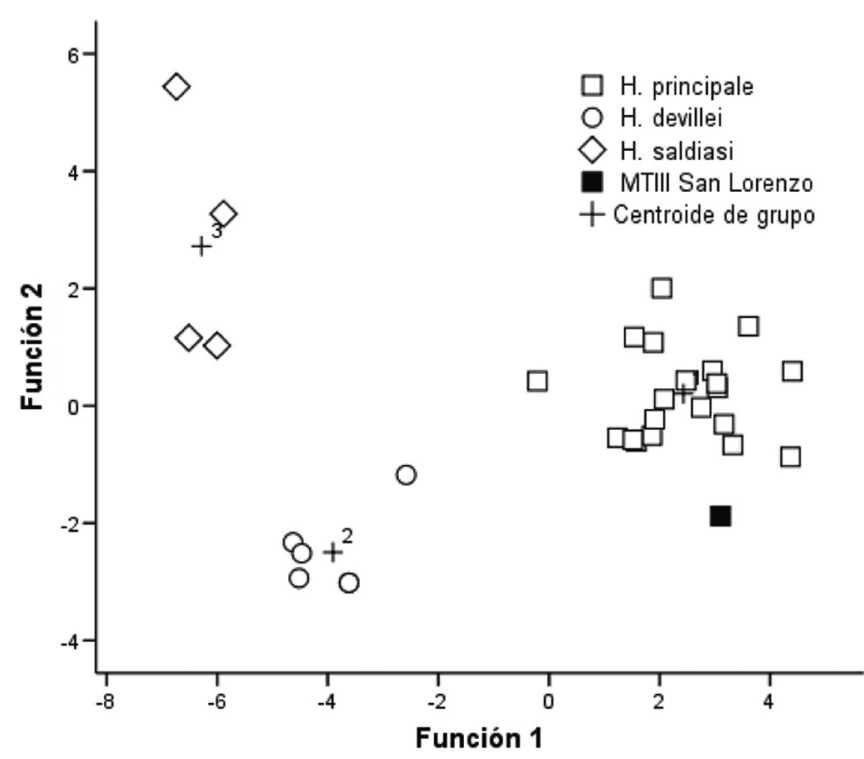

Figura 6. Análisis discriminante del MCIII de Hippidion principale de San Lorenzo (Puerto General San Martín, bajada del Quebracho, Santa Fé, Argentina) en relación con 35 metatarsianos de distintas especies de Hippidion de América del Sur. 1, 2 y 3 son los centroides. Hippidion principale MCIII discriminant analysis from San Lorenzo (General San Martín Port, Quebracho slope, Santa Fe, Argentina) compared with 35 metatarsians of other Hippidion species from South America. 1, 2 and 3 are centroids.

al Bonaerense en la localidad de Mercedes en la provincia de Buenos Aires (Alberdi \& Prado, 2004) y en el sitio arqueológico Arroyo Seco 2 en la localidad de Tres Arroyos (Politis et al., 1987).

En las facies conglomeráticas basales de la Formación Sopas (Edad Lujanense) se ha obtenido por termoluminiscencia (TL) una edad de $43 \pm 3,6 \mathrm{ka}$ a $58,3 \pm 7,4 \mathrm{ka}$ (Ubilla, 2004). En Argentina, los niveles correlacionables se encuentran en el tramo basal de la cuenca del río Carcarañá, que en la provincia de Santa Fé desemboca en el río Paraná. Estos niveles, asignados a la Formación Carcarañá, han sido datados mediante TL en una edad entre $45,6 \pm 1,9$ ka y $52,3 \pm 1,2 \mathrm{ka}$ (Krohling, 1999).

Por su parte, Toledo (2005) estima una edad similar para el piso Bonaerense descripto en las barrancas del río Luján, muy próximo a la ciudad de Mercedes.

La otra localidad con coexistencia de ambas especies es el sitio Arroyo Seco 2, cuyas dataciones lo ubican en $10 \mathrm{ka}$.

La sección estratigráfica analizada en San Lorenzo no cuenta con dataciones radiométricas o estudios paleomagnéticos que permitan calibrar con precisión la edad de los depósitos portadores de équidos. Sobre la base de las correlaciones estratigráficas, los restos hallados corresponderían al lapso entre los $128 \mathrm{ka}$ (el inicio del Pleistoceno Superior, estadio isotópico $5 \mathrm{e}$ ), como edad máxima, hasta momentos anteriores al comienzo del estadio isotópico 2e (28 ka).

Esta inferencia se ajusta a la interpretación paleoclimática, ya que esta asociación de caballos es compatible con condiciones interglaciales. Si la fauna es referible al Lujanense [entre otras evidencias por la presencia de Equus (Amerhippus) neogeus], dichas condiciones deben corresponder al último interglacial.

Diversas evidencias permiten inferir que el Lujanense comienza con condiciones interglaciales (EI 5e, $130 \mathrm{ka}$ AP, base del Pleistoceno superior), faunísticamente reconocibles (Pardiñas et al., 2004). La parte final del Lujanense se desarrolló coincidentemente con el Último Máximo Glacial (18 ka AP) e incluye el último avance glacial (13 -11 ka AP), predominando en este lapso una fauna adaptada a condiciones áridas y frías (Tonni et al., 1999, 2003). Al finalizar el Pleistoceno ( $c a .10$ ka AP) condiciones cálidas y húmedas generan un episodio pedogenético (Tonni et al., 2001).

En suma, la presencia de ambas especies de caballos se corresponde con intervalos más cálidos y húmedos que 
se vinculan con los cambios biogeográficos vinculados a la alternancia de ambientes glaciales e interglaciales durante el Pleistoceno

\section{AGRADECIMIENTOS}

Queremos agradecer especialmente a Juan Nobile responsable del Museo de Historia y Galería de Arte del Centro Cultural de San Lorenzo por haber puesto a nuestra disposición este material y todas las facilidades para su estudio y a Pedro Mondori que recuperó los ejemplares. También queremos agradecer a Esperanza Cerdeño y Dolores Pesquero sus comentarios críticos que han mejorado considerablemente el manuscrito. Este trabajo se ha realizado en el marco de los Proyectos CGL200400400/BTE y CGL2007-60790/BTE de la DGCYT, España, y las Subvenciones de la Universidad Nacional del Centro de la Provincia de Buenos Aires y el Proyecto PIP-02773, CONICET y ANPCYT PICTO 04-11503, Argentina.

\section{REFERENCIAS}

Alberdi, M.T. \& Frassinetti, D. 2000. Presencia de Hippidion y Equus (Amerhippus) (Mammalia, Perissodactyla) y su distribución en el Pleistoceno Superior de Chile. Estudios Geológicos, 56, 279-290.

Alberdi, M.T. \& Prado, J.L. 1992. El registro de Hippidion Owen, 1869 y Equus (Amerhippus) Hoffstetter, 1950 (Mammalia, Perissodactyla) en América del Sur. Ameghiniana, 29, 265-284.

Alberdi, M.T. \& Prado, J.L. 1993. Review of the genus Hippidion Owen, 1869 (Mammalia; Perissodactyla) from the Pleistocene of South America. Zoological Journal of the Linnean Society, 108, 1-22.

Alberdi, M.T. \& Prado, J.L. 2004. Caballos fósiles de América del Sur. Una historia de tres millones de años. INCUAPA Serie Monográfica, 3, 1-269.

Alberdi, M.T. \& Prieto, A. 2000. Hippidion (Mammalia, Perissodactyla) de las Cuevas de las provincias de Magallanes y Tierra de Fuego. Anales del Instituto de la Patagonia, Serie Ciencias Humanas (Chile), 28, 147-171.

Alberdi, M.T., Fernández, J., Menegaz, A.N. \& Prado, J.L. 1986. Hippidion Owen 1869 (Mammalia, Perissodactyla) en sedimentos del Pleistoceno tardio de la localidad Barro Negro (Jujuy, Argentina). Estudios geológicos, 42, 487-493.

Alberdi, M.T., Menegaz, A.N. \& Prado, J.L. 1987. Formas terminales de Hippidion (Mammalia, Perissodactyla) de los yacimientos del Pleistoceno tardío - Holoceno de la Patagonia (Argentina y Chile). Estudios Geológicos, 43, 107-115.

Alberdi, M.T., Menegaz, A.N., Prado, J.L. \& Tonni, E.P. 1989. La fauna local de Quequén Salado-Indio Rico (Pleistoceno tardío) de la provincia de Buenos Aires, Argentina. Aspectos paleoambientales y biostratigráficos. Ameghiniana, 25, 225-236.

Alberdi, M.T., Prado, J.L. \& Miotti, L. 2001a. Hippidion saldiasi Roth, 1899 (Mammalia, Perissodactyla) at the Piedra
Museo Site (Patagonia): their implication for the regional economy and environmental. Journal of Archaeological Science, 28, 411-419.

Alberdi, M.T., Zárate, M. \& Prado, J.L. 2001b. Presencia de Hippidion principale en los acantilados costeros de Mar del Plata (Argentina). Revista Española de Paleontología, 16, 1-7.

Alberdi, M.T., Cartelle, C. \& Prado, J.L. 2003. El registro Pleistoceno de Equus (Amerhippus) e Hippidion (Mammalia, Perissodactyla) de Brasil. Consideraciones paleoecológicas y biogeográficas. Ameghiniana, 40, 173-196.

Alberdi, M.T., Prado, J.L. \& Favier-Dubois, C.M. 2006. Nuevo registro de Hippidion principale (Mammalia, Perissodactyla) del Pleistoceno de Mar del Sur, Argentina. Revista Española de Paleontología, 21, 105-113.

Alberdi, M.T., Prado, J.L., López, P., Labarca, R. \& Martínez, I. 2007. Hippidion saldiasi Roth, 1899 (Mammalia, Perissodactyla) en el Pleistoceno tardío de Calama, norte de Chile. Revista Chilena de Historia Natural, 80, 157-171.

Alberdi, M.T., Cerdeño, E. \& Prado, J.L. 2008. Stegomastodon platensis (Proboscidea, Gomphotheriidae) en el Pleistoceno de Santiago del Estero, Argentina. Ameghiniana, 45, 257-271.

Ameghino, F. 1889. Contribución al conocimiento de los mamíferos fósiles de la República Argentina. Actas de la Academia Nacional de Ciencias de Córdoba, 6, 1-1027.

Ameghino, F. 1904. Los Mamíferos fósiles de la República Argentina. Obras Completas. Editado por A. Torcelli, Buenos Aires, 198-298.

Branco, W. 1883. Ueber eine fossile Säugethier-Fauna von Punin bei Riobamba in Ecuador. II: Beschreibung der Fauna. Palaeontologische Abhandlunge, 1, 57-204.

Castellanos, A. 1924. Contribución al estudio de la Paleoantropología argentina (Restos descubiertos en la laguna Melincué, provincia de Santa Fé). Revista de la Universidad Nacional de Córdoba, 10-11-12, 1-34.

Castellanos, A. 1938. El subsuelo de Rosario. Anales de la Sociedad Científica Argentina, 127, 3-13.

Castellanos A., 1943. El Preensenadense ies un horizonte geológico o una facies? Publicaciones del Instituto de Fisiografía y Geología, 18, 1-85.

Cione, A.L. \& Tonni, E.P. 1999. Biostratigraphy and chronological scale of upper-most Cenozoic in the Pampean Area, Argentina. Quaternary of South America and Antarctica Peninsula, 12, 23-51.

Daniel, H. 1948. Nociones de Geología y Prehistoria de Colombia. P. Imprenta Bedout FF.SS.CC., Medellín, 360 pp.

Darwin, C. 1846. Geological observations. D. Appleton Co., London, $648 \mathrm{pp}$.

De Carles, E. 1912. Relaciones acerca de los yacimientos fosilíferos del arroyo Frías y sedimentos de la barrancas del río Paraná al norte y sur de Santa Fé. Anales Museo Nacional de Historia Natural de Buenos Aires, 23, 245-252.

D'Orbigny A. 1842-1844. Voyage dans l'Amérique Méridionale. Paleontologie. Bertrand edit., Paris \& Strassbourg, 3 (4), 1-152.

Eisenmann, V., Alberdi, M.T., De Giuli, G. \& Staesche, U. 1988. Collected papers after the "New York International 
Hipparion Conference, 1981”. In: Studying fossil horses, 1, Methodology (Eds. M. Woodbrune \& P. Sondaar). E.J. Brill, Leiden, 1-72.

Fidalgo F., De Francesco F. \& Pacual R., 1975. Geología superficial de la llanura bonaerense. Relatorio: Geología de la Provincia de Buenos Aires. Sexto Congreso Geológico Argentino, 103-138.

Frenguelli, J. 1928. Sobre un perfil geológico del río Carcarañá. Anales Facultad de Ciencias de la Educación de Paraná, 3, 101-130.

Frenguelli, J. 1932. Perfiles geológicos de las márgenes del río Salado (Santa Fe). Publicaciones Museo Antropológico y Etnográfico, ser. A 2, 83-97.

Gervais, P. 1855. Recherches sur les Mammifères fossiles de l'Amérique méridionale. Chez P. Bertrand, Libraireediteur, Paris, 1-63.

González Bonorino F. 1965. Mineralogía de las fracciones arcilla y limo del Pampeano en el área de la Ciudad de Buenos Aires y su significado estratigráfico y sedimentológico. Revista de la Asociación Geológica Argentina, 20, 67-150.

Gray, J.E. 1821. On the natural arrangement of vertebrate animals. London Medical Reposit Review, 15, 296-310.

Hoffstetter, R. 1950. Algunas observaciones sobre los caballos fósiles de la América del Sur. Amerhippus gen. nov. Boletín Informaciones Científicas Nacionales, 3, 426-454.

Iriondo, M. 1991. El holoceno en el litoral Santa Fé. Comunicaciones del Museo Provincial de Ciencias Naturales Florentino Ameghino, Santa Fé, 3, 1-38.

Krohling, D. 1999. Upper Quaternary geology of the lower Carcarana Basin, North Pampa, Argentina. Quaternary International, 57/58, 135-148.

Lund, P.W. 1840. Nouvelles recherches sur la faune fossile du Brésil. Annales Science Naturalles, 13, 310-319.

Lund, P.W. 1846. Meddlelse af det Udbytte de I 1844 undersögte Knoglehuler Have avgivet til hundskaben om Brasiliens Dyreverden för sidste Jordomvaeltning. Det köngelige Danske Videnskabernes Selskabs Naturvidenskabelige og Mathematiske Afhandlinger, 12, 57-94.

Miño Boilini, A.R., Cerdeño, E. \& Bond, M. 2006. Revisión del género Toxodon Owen, 1837 (Notoungulata: Toxodontidae) en el Pleistoceno de las provincias de Corrientes, Chaco y Santa Fé, Argentina. Revista Española de Paleontología, 21, 93-103.

Owen, R. 1840. The Zoology of The Voyage of H.M.S. Beagle under the command of Captain Fitzroy, R.N., during the years 1832 to 1836. Part I.- Fossil Mammalia. Ed. \& superv. C. Darwin, 108.

Owen, R. 1848. Description of teeth and portions of jaws of two extinct anthracotherioid quadrupeds ... discovered ... in the Eoecene deposits on the NW coast of the Isle of Wight. Quarterly Journal Geological Society, London, 4, 103-141.

Owen, R. 1869. On Fossil Remains of Equines from Central and South America referable to Equus conversidens, Ow., Equus tau, Ow., and Equus arcidens, Ow. Philosophical Transactions Royal Society, London, 159, 559-573.

Pardiñas, U.F.J., Cione, A.L., San Cristóbal, J., Verzi, D.H. \& Tonni, E.P. 2004. A new interglacial continental vertebrate assemblage in central-eastern Argentina. Current Research in the Pleistocene, 21, 111-112.

Parent, H., Polare, M. \& Lattuca, F. 2002. Estratigrafía. In: Geología y Geomorfología de la Cuenca del Arroyo del Medio, Provincia Santa Fé (Argentina) (Eds. O.A. Albert, F. Lattuca \& H. Parent). Boletín del Instituto de Fisiografía y Geología, 72, 1-14.

Politis, G., Tonni, E.P., Hidalgo, F., Salemme, M. \& Leo Guzmán, L. 1987. Man and Pleistocene Megamammals in the Argentine Pampa: Site 2 at Arroyo Seco. Current Research in the Pleistocene, 4, 159-161.

Porta, J. de 1960. Los équidos fósiles de la sabana de Bogotá. Boletín de Geología. Universidad Industrial de Santander, Colombia, 4, 51-78.

Prado, J.L. \& Alberdi, M.T. 1994. A Quantitative Review of the Horse "Equus" from South America. Palaeontology, 37, 459-481.

Prado, J.L. \& Alberdi, M.T. 1996. A cladistic analysis of the Horses of the tribe Equini. Palaeontology, 39, 663-680.

Prado, J.L., Alberdi, M.T. \& Reguero, M.A. 1998. El registro más antiguo de Hippidion Owen, 1869 (Mammalia, Perissodactyla) en América del Sur. Estudios Geológicos, 54, 85-91.

Prado, J.L., Alberdi, M.T., Martínez, G. \& Gutiérrez, M.A. 2005. Equus (Amerhippus) neogeus Lund, 1840 (Equidae, Perissodactyla) at Paso Otero 5 site (Argentina): Its implications for the extinction of the South American horse. Neues Jahrbuch für Geologie und Paläontologie Mh., 2005, 449-468.

Rincón, A.D., Alberdi, M.T. \& Prado, J.L. 2006. Nuevo registro de Equus (Amerhippus) santaeelenae (Mammalia, Perissodactyla) del pozo de asfalto de Inciarte (Pleistoceno Superior), estado Zulia, Venezuela. Ameghiniana, 43, 529-538

Roth, S. 1899. El mamífero misterioso de la Patagonia Grypotherium domesticum. II. Descripción de los restos encontrados en la Caverna de Última Esperanza. Revista Museo de La Plata, 9, 421-453.

Spillmann, F. 1938. Die fossilen Pferde Ekuadors der Gattung Neohippus. Palaeobiologica, 6, 372-393.

Toledo, M.J. 2005. Secuencias pleistocenas «lujanenses» en su sección tipo: Primeras dataciones ${ }^{14} \mathrm{C}$ e implicancias estratigráficas, arqueológicas e históricas, Luján - Jáuregui, provincia de Buenos Aires. Revista Asociación Geológica Argentina, 60, 417-424.

Tonni E.P., Nabel P., Cione A.L., Etchichury M., Tófalo R., Scillato Yané G., San Cristóbal J., Carlini A. \& Vargas D. 1999. The Ensenada and Buenos Aires formations (Pleistocene) in a quarry near La Plata, Argentina. Journal of South American Earth Sciences, 12, 273-291.

Tonni, E.P., Cione, A.L. \& Figini, A.J. 2001. Chronology of Holocene pedogenetic events in the Pampean area of Argentina. Current Research in the Pleistocene, 18, 124-127.

Tonni, E.P., Huarte, R.A., Carbonari, J.E. \& Figini, A.J. 2003. New radiocarbon chronology for the Guerrero Member of the Luján Formation (Buenos Aires, Argentina): palaeoclimatic significance. Quaternary International, 109-110, 45-48. 
Ubilla, M. 2004. Mammalian biostratigraphy of Pleistocene fluvial deposits in northern Uruguay, South America. Proceedings of the Geologists' Association, 115, 347-357.

Ubilla, M. \& Alberdi, M.T. 1990. Hippidion sp. (Mammalia, Perissodactyla, Equidae) en sedimentos del Pleistoceno Superior del Uruguay (Edad Mamífero Lujanense). Estudios Geológicos, 46, 453-464.

Ubilla, M., Perea, D., Goso Aguilar, C. \& Lorenzo, N. 2004. Late Pleistocene vertebrales from northern Urugua: tools for biostratigraphic, climatic and environmental reconstruction. Quaternary International, 114, 129-142.
Zurita, A.E., Carlini, A. A., Scillato-Yané, G.J., Parent, H., Nieto, M. C. \& Franco, D.C. 2002. Un nuevo yacimiento de mamíferos pleistocenos en el arroyo El Tapialito, Reconquista, provincia de Santa Fé. Consideraciones bioestratigráficas y paleoambientales. $8^{\circ}$ Congreso Argentino de Paleontología y Bioestratigrafía, Corrientes, Resúmenes, 57.

Manuscrito recibido: 21 de Abril, 2008 Manuscrito aceptado: 10 de Septiembre, 2008 